9-17-1992

\title{
Grounding of Space Structures
}

\author{
Paul A. Bosela \\ Cleveland State University, p.bosela@csuohio.edu \\ D. G. Fertis \\ University of Akron \\ F. J. Shaker \\ NASA Lewis Research Center
}

Follow this and additional works at: https://engagedscholarship.csuohio.edu/encee_facpub

Part of the Structures and Materials Commons

How does access to this work benefit you? Let us know!

\section{Publisher's Statement}

NOTICE: this is the author's version of a work that was accepted for publication in Computers \& Structures. Changes resulting from the publishing process, such as peer review, editing, corrections, structural formatting, and other quality control mechanisms may not be reflected in this document. Changes may have been made to this work since it was submitted for publication. A definitive version was subsequently published in Computers \& Structures, 45, 1, (09-17-1992); 10.1016/0045-7949(92)90351-Y

\section{Original Citation}

Bosela, P. A., Fertis, D. G., and Shaker, F. J. (1992). "Grounding of space structures." Computers and Structures, 45(1), 143-153.

This Article is brought to you for free and open access by the Civil and Environmental Engineering at EngagedScholarship@CSU. It has been accepted for inclusion in Civil and Environmental Engineering Faculty Publications by an authorized administrator of EngagedScholarship@CSU. For more information, please contact library.es@csuohio.edu. 


\title{
GROUNDING OF SPACE STRUCTURES
}

\author{
P. A. Bosela $\dagger$, D. G. Fertis $\ddagger$ and F. J. Shaker§ \\ † Department of Engineering Technology, Cleveland State University, Cleveland, OH 44115, U.S.A. \\ $\ddagger$ Department of Civil Engineering, University of Akron, Akron, $\mathrm{OH} 44325$, U.S.A. \\ § Engineering Directorate, Structural Systems Division, Dynamics Branch, \\ NASA Lewis Research Center, Cleveland, Ohio, U.S.A.
}

\begin{abstract}
Space structures, such as the Space Station solar arrays, must be extremely light-weight, flexible structures. Accurate prediction of the natural frequencies and mode shapes is essential for determining the structural adequacy of components, and designing a controls system. The tension pre-load in the 'blanket' of photovoltaic solar collectors, and the free/free boundary conditions of a structure in space, causes serious reservations on the use of standard finite element techniques of solution. In particular, a phenomenon known as 'grounding', or false stiffening, of the stiffness matrix occurs during rigid body rotation. This paper examines the grounding phenomenon in detail. Numerous stiffness matrices developed by others are examined for rigid body rotation capability, and found lacking. A force imbalance inherent in the formulations examined is the likely cause of the grounding problem, suggesting the need for a directed force formulation.
\end{abstract}

\section{NOTATION}

\begin{tabular}{|c|c|}
\hline$A$ & area $\left(\mathrm{in}^{2}\right)$ \\
\hline $\begin{array}{l}{\left[A_{1}\right]} \\
B\end{array}$ & $\begin{array}{l}\text { Paz's second order mass-geometrical matrix } \\
/(P / E I R)\end{array}$ \\
\hline$b_{1}, b_{2}, b_{3}, b_{4}$ & $\begin{array}{l}\text { constants in polynomial expression for Mar- } \\
\text { cal's initial displacement matrices }\end{array}$ \\
\hline$E$ & Young's modulus (psi) \\
\hline$G$ & shear modulus \\
\hline$\left[G_{0}\right]$ & Paz's consistent geometric stiffness matrix \\
\hline$\left[G_{1}\right]$ & Paz's second order geometric matrix \\
\hline$I^{*}$ & $\begin{array}{l}\text { moment of inert } \\
I\left(1+P\left(K^{\prime} A G\right)\right.\end{array}$ \\
\hline$K^{\prime} A G$ & beam shear rigidity \\
\hline & shear factor \\
\hline$[K]$ & stiffness matrix \\
\hline$\left[K_{e}\right]$ & elastic stiffness matrix \\
\hline & $\begin{array}{l}\text { elastic stiftness matrix for a Timoshenko } \\
\text { beam }\end{array}$ \\
\hline
\end{tabular}

$\left[K_{g}\right] \quad$ geometric stiffness matrix

$\left[K_{g}\right]_{3 \text {-node }}$ geometric stiffness matrix for a three-node beam

$\left[K_{N C}\right] \quad$ Argyris load correction matrix for a tangent follower force

$L \quad$ beam length

$\left[M_{0}\right] \quad$ Paz's first order mass matrix

$\left[M_{1}\right]$

$[M]$

$m$

$\left[\tilde{N}_{1}\right]$

$\left[\tilde{N}_{2}\right]$

$\left[N_{1}\right]$

$\left[N_{2}\right]$

$P$

$P^{\prime}$

$R$

$\left\{\boldsymbol{R}_{i}\right\}$

$\left[R_{0}\right]$

$[S]$

$\left\{U_{T X}\right\}$

$\left\{U_{T Y}\right\}$

$\left\{U_{R B R}\right\}$
$\{U\}$

$u$

$v$

$z$

$\alpha$

$\beta$

$\boldsymbol{\phi}$

$\phi\left(\beta^{4}\right)$

$\mathbf{\Omega}$

\author{
displacement or mode shape vectors \\ axial displacement \\ transverse displacement \\ $\sin (B L)(2 \tan B L / 2-B L R)$ \\ $(B L)$ \\ half the angle of rigid body rotation \\ shear factor $=12 E I / L^{2} K^{\prime} A G$ for Timo- \\ shenko beam \\ a function of $\beta^{4}$ \\ rotation angle \\ frequency $(\mathrm{rad} / \mathrm{sec})$
}

\section{INTRODUCTION}

In order to be cost-effective, space structures must be extremely light-weight, and subsequently, very flexible structures. The power system for Space Station Freedom is such a structure. Each array consists of a deployable truss mast and a split 'blanket' of photovoltaic solar collectors. The solar arrays are deployed in orbit, and the blanket is stretched into position as the mast is extended during deployment. Geometric stiffness due to the tension pre-load in the blanket makes this an interesting non-linear problem.

The space station will be subjected to various dynamic loads during shuttle docking, solar tracking, attitude adjustment, etc. Accurate prediction of the natural frequencies and mode shapes of the space station components, including the solar arrays, is critical for determining the structural adequacy of the components, and for designing a dynamic controls system.

This paper has the following objectives:

1. To examine in detail the 'grounding' phenomenon associated with rigid body rotation of a preloaded beam in space.

2. To examine beam geometric stiffness matrices developed by others with respect to rigid body motion capabilities. 
Table 1. Comparison of finite element method versus exact solution for a beam in tension

$$
\begin{aligned}
& A=48 \mathrm{in}^{2} \\
& E=30 \times 10^{6} \mathrm{ps} 1 \\
& I=1000 \mathrm{in}^{4} \\
& \mathrm{P}=10.000 .000 \mathrm{lb} \\
& L=100 \mathrm{IN}
\end{aligned}
$$

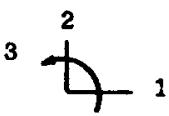

Pin/Roller

\begin{tabular}{|c|cccc|c|c|}
\hline & \multicolumn{3}{|c|}{ number of elements } & $*$ error & Figorous sol \\
freq & 1 & 2 & 4 & 8 & & Fertis [3] \\
\hline 1 & 1142 & 1056 & 1053 & 1053 & 3.5 & 1043 \\
2 & 3501 & 3257 & 3195 & 3180 & axial & \\
3 & 4758 & 4180 & 3807 & 3794 & 4.0 & 3647 \\
4 & & 10291 & 8494 & 8357 & 9.0 & 7669 \\
\hline
\end{tabular}

Free/Free Beam

\begin{tabular}{|c|cccc|c|c|}
\hline freq & \multicolumn{2}{|c|}{ number of elements } & * error & $\begin{array}{c}\text { rigorous sol } \\
\text { Boselatshaker } \\
{[4]}\end{array}$ \\
\hline 1 & 1 & 2 & 4 & 8 & 0 & 0 \\
2 & 0 & 0 & 0 & 0 & 0 & 0 \\
3 & 580 & 580 & 579 & 579 & $\infty$ & 0 \\
4 & 2798 & 2383 & 2381 & 2378 & 15.0 & 2017 \\
5 & 7001 & 6725 & 5991 & 5958 & 4.7 & 5725 \\
\hline
\end{tabular}

\section{LIMITATIONS OF CURRENT METHODOLOGY}

Most structural systems are rigidly attached to supports at either or both ends. In order for any movement to occur, the structure must deform, and internal strain energy is developed. Space structures, on the other hand, are not rigidly attached to the ground. Instead, they are free to move as rigid bodies as well as to deform.

Complex structures are generally analyzed using finite element computer programs which solve the dynamic equations of motion using matrix analysis techniques. The equations of motion are set up in the form of the generalized eigenvalue problem

$$
\left[[K]-\Omega_{i}^{2}[M]\right]\left\{u_{i}\right\}=\left\{R_{i}\right\},
$$

where $[K]$ is the global stiffness matrix, $[M]$ is the global mass matrix, $\Omega_{i}$ are the natural frequencies of vibration, $\left\{\boldsymbol{u}_{i}\right\}$ are the displacement or mode shape vectors, and $\left\{\boldsymbol{R}_{i}\right\}$ are the forces.
Using that basis, rigid body modes are the eigenvectors associated with zero frequencies of vibrations (eigenvalues).

Current methodology utilizes MSC/NASTRAN Solution 64 to generate the tangential stiffness matrix for the deployed array, storing this matrix in a database, then using this matrix in Solution $63 \mathrm{dy}-$ namic analysis, to obtain the frequencies of vibration. As a routine check of the model, the global stiffness matrix is multiplied with a matrix of the rigid body modes to determine whether any pseudo-forces occur. (Whether strain energy has developed.) Since no internal stresses should occur during rigid body motion, the generation of pseudo-forces indicates that an internal 'grounding', or false stiffening, of the system occurs, due to errors or deficiencies in the finite element model.

It was found that the global stiffness matrix does not possess rigid body rotation capabilities. In order to predict the dynamic response of the structure, a Craig-Bampton substructuring scheme is used. However, certain erroneous non-zero terms appear in the 
null set of the partitioned matrices due to the grounding effect. They must be zeroed out, and the missing rigid body modes appended to the matrix, in order to more accurately predict the dynamic response [1].

The author idealized the problem as a free/free beam in tension, and found that the pseudo-forces are developed at the element level due to limitations inherent in the geometric stiffness matrices currently in acceptable use. In particular, the geometric stiffness matrices for the beam element lack the capability for rigid body rotations, especially when the rotations are large.

The geometric (initial stress) stiffness matrices in current use developed from a Bernoulli-Euler formu- convergence to the missing zero frequency in the dynamics problem of the pre-loaded beam with free/free boundary conditions. In addition, higher frequencies may be significantly in error. Table 1 compares the finite element solution for a pretensioned beam with pinned/roller and free/free boundary conditions.

\section{ELASTIC STIFFNESS MATRIX}

The elastic stiffness matrix for a two-node Bernoulli beam is

$$
\left[K_{e}\right]=\frac{E I}{L^{3}}\left[\begin{array}{cccccc}
A L^{2} / I & 0 & 0 & -A L^{2} / I & 0 & 0 \\
0 & 12 & 6 L & 0 & -12 & 6 L \\
0 & 6 L & 4 L^{2} & 0 & -6 L & 2 L^{2} \\
-A L^{2} / I & 0 & 0 & A L^{2} / I & 0 & 0 \\
0 & -12 & -6 L & 0 & 12 & -6 L \\
0 & 6 L & 2 L^{2} & 0 & -6 L & 4 L^{2}
\end{array}\right] .
$$

The $\left[K_{e}\right]$ matrix must possess the capacity of a full set of rigid body modes. In other words, the element must be able to both translate and rotate without developing stresses (see Fig. 1). lation have been shown to provide accetable results for most static displacement and buckling problems, provided a sufficient number of elements are used [2]. However, refinement of the mesh does not produce

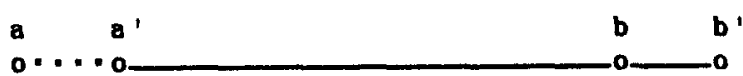

a. Rigid Body Transiation In Axial Direction

$$
\left(U_{T X}\right)=[1,0,0,1,0,0]^{T}
$$

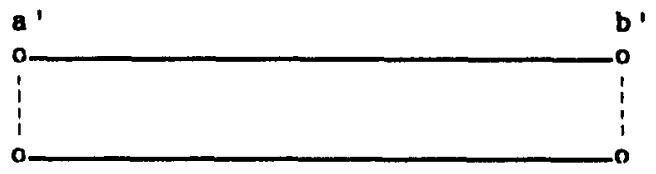

a

b

b. Rigid Body Translation in Transverse Direction

$$
\left\{U_{T Y}\right\}=[0,1,0,0,1,0]^{T}
$$

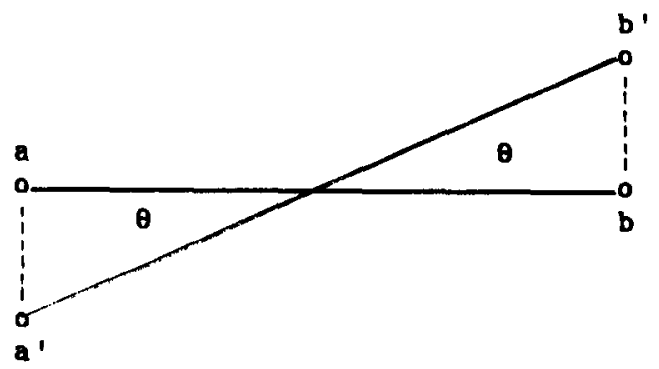

c. Rigid Body Rotation

$$
\left\{U_{R B R}\right\}=\theta[0,-L / 2,1,0, L / 2,1]^{T}
$$

Fig. 1. Rigid body modes. 
Note that in Fig. 1(c) that the rotation is considered to be relatively small, such that the displacement in the axial direction due to the rotation is negligible.

Multiplying $\left[K_{e}\right] \times[$ rigid body mode $]$

$$
=[0,0,0,0,0,0]
$$

holds for all three modes. Hence, $\left[K_{e}\right]$ possesses all the required rigid body mode capabilities.

Another way of determining whether $\left[K_{e}\right]$ possesses all the rigid body mode capabilities is to solve the dynamic analysis of the beam with free/free boundary conditions. This was done [6] using the finite element dynamics algorithm in the computer program NLFINITE.FOR (see the Appendix). The results were three zero eigenvalues and corresponding rigid body mode shapes. stiffness, or initial stress stiffness matrix. Various formulations of the geometric stiffness matrix have been developed.

When the Hermitian interpolating polynomials (used to derive the $\left[K_{e}\right]$ matrix) are used in deriving the geometric stiffness coefficients, the resulting $\left[K_{g}\right]$ is referred to as the consistent geometric stiffness matrix (Bernoulli beam geometric stiffness)

$$
\begin{aligned}
{\left[K_{g}\right]=} & P /(30 L) \\
& \times\left[\begin{array}{cccccc}
0 & 0 & 0 & 0 & 0 & 0 \\
0 & 36 & 3 L & 0 & -36 & 3 L \\
0 & 3 L & 4 L^{2} & 0 & -3 L & -L^{2} \\
0 & 0 & 0 & 0 & 0 & 0 \\
0 & -36 & -3 L & 0 & 36 & -3 L \\
0 & 3 L & -L^{2} & 0 & -3 L & 4 L^{2}
\end{array}\right]
\end{aligned}
$$

Application of the rigid body modes to $\left[K_{g}\right]$ results in

$$
\left[K_{g}\right] \times\left[\begin{array}{ccc}
1 & 0 & 0 \\
0 & 1 & -L \theta / 2 \\
0 & 0 & \theta \\
1 & 0 & 0 \\
0 & 1 & L \theta / 2 \\
0 & 0 & \theta
\end{array}\right]=\left[\begin{array}{ccc}
0 & 0 & 0 \\
0 & 0 & -P \theta \\
0 & 0 & 0 \\
0 & 0 & 0 \\
0 & 0 & P \theta \\
0 & 0 & 0
\end{array}\right]
$$

Another beam stiffness matrix which incorporates shear effects is referred to as a Timoshenko beam. The elastic stiffness matrix for a Timoshenko beam is
The terms $\pm P \theta$ are fictitious forces generated during the rigid body rotation. Similarly, dynamic analysis, using NLFINITE.FOR, yields only two zero eigenvalues for the free/free beam in tension,

$$
\left[K_{e}\right]=\frac{E I(1 /(1+\Phi))}{L^{3}}\left[\begin{array}{cccccc}
A L^{2}(1+\Phi) / I & 0 & 0 & -A L^{2}(1+\Phi) / I & 0 & 0 \\
0 & 12 & 6 L & 0 & -12 & 6 L \\
0 & 6 L & (4+\Phi) L^{2} & 0 & -6 L & (2-\Phi) L^{2} \\
-A L^{2}(1+\Phi) / I & 0 & 0 & A L^{2}(1+\Phi) / I & 0 & 0 \\
0 & -12 & -6 L & 0 & 12 & -6 L \\
0 & 6 L & (2-\Phi) L^{2} & 0 & -6 L & (4+\Phi) L^{2}
\end{array}\right]
$$

where $\Phi=12 E I /\left(L 2 K^{\prime} A G\right)$, which corrects for shear deformation. As $K^{\prime} A G$ becomes very large, $\Phi \approx 0$, and $\left[K_{e}\right]_{T}=\left[K_{e}\right]$. The Timoshenko elastic stiffness also possesses a full set of rigid body modes.

A major difference in the Timoshenko approach is that the bending rotation is considered independently in the derivation, not simply the derivative of the displacement equation, as is done in the Bernoulli derivation.

\section{GEOMETRIC STIFFNESS MATRIX DEVELOPMENT}

The presence of an axial force introduces additional stiffness terms, resulting in the geometric corresponding to axial and transverse rigid body translations only.

Various formulations have been used for establishing the geometric stiffness matrices from the static displacement problem. Martin [7] used a strain energy formulation with interpolating polynomials. Clough and Penzien [8] used minimization of the potential function with the Hermitian polynomials. Both approaches yield a consistent geometric stiffness matrix, which lacks rigid body rotation capability, as was previously demonstrated. 
The first author followed Martin's methodology in developing a three-node beam geometric stiffness matrix [9]. The following matrix was obtained

$$
P\left[\begin{array}{ccccccccc}
\frac{7}{3 L} & & & & & & & \\
0 & \frac{18272}{105 L} & & & & & & \\
0 & \frac{3469}{105} & \frac{659 L}{105} & & & & \text { Symmetric } & \\
\frac{-20}{3 L} & 0 & 0 & \frac{64}{3 L} & & & & & \\
0 & \frac{-22096}{105 L} & \frac{-4208}{105} & 0 & \frac{27292}{105 L} & & & & \\
0 & \frac{304}{5} & \frac{23 L}{2} & 0 & -72 & \frac{109 L}{5} & & & \\
\frac{13}{3 L} & 0 & 0 & \frac{-44}{3 L} & 0 & 0 & \frac{31}{3 L} & & \\
0 & \frac{3824}{105 L} & \frac{739}{105} & 0 & \frac{-5296}{105 L} & \frac{56}{5} & 0 & \frac{1472}{105 L} & \\
0 & \frac{-3469}{105} & \frac{-659 L}{105} & 0 & \frac{4208}{105} & \frac{-23 L}{2} & 0 & \frac{-739}{105} & \frac{659 L}{105}
\end{array}\right]
$$

By inspection, $\left[K_{g}\right]_{3-\text { node }}$ has two rigid body translational capabilities. The exact rigid body rotation vector is

$$
\begin{array}{r}
{[L(1-\cos (2 \beta)) / 2,-L \sin (2 \beta) / 2,2 \beta, 0,0,2 \beta,} \\
-L(1-\cos (2 \beta)) / 2, L \sin (2 \beta) / 2,2 \beta]^{T},
\end{array}
$$

where $\beta$ is half the angle of rotation.

If this vector is expanded in power series form, upon retaining the first two terms, and factoring out $\beta L$, one obtains

$$
\begin{array}{r}
\left\{U_{R B R}\right\}^{T}=\left[\beta-\beta^{3} / 3,-1+2 \beta^{2} / 3,2 / L, 0,0,2 / L,\right. \\
\left.-\beta+\beta^{3} / 3,1-2 \beta^{2} / 3,2 / L\right]
\end{array}
$$

Multiplying $\left[K_{g}\right]$ by $\left\{U_{R B R}\right\}$ yields

$$
\begin{gathered}
{\left[-2 \beta^{2}, 91.73 \beta^{3}-16 \beta, L\left(17.3 \beta^{3}-3 \beta\right), 8 \beta^{2},\right.} \\
16 \beta-106.7 \beta^{3}, L\left(33.01 \beta^{3}-6 \beta\right), \\
\left.-6 \beta^{2}, 14.93 \beta^{3}, L\left(3 \beta-17.33 \beta^{3}\right)\right] .
\end{gathered}
$$

which contains numerous non-zero terms. Hence, $\left[K_{g}\right]_{3-\text { node }}$ does not possess rigid body rotation capability.

Saunders [10] solves for the exact solution of the differential equations using a Timoshenko approach, then expands his 'exact' stiffness matrix in a power series solution, obtaining a series of matrices of increasing order.

Saunders 'exact' stiffness matrix is

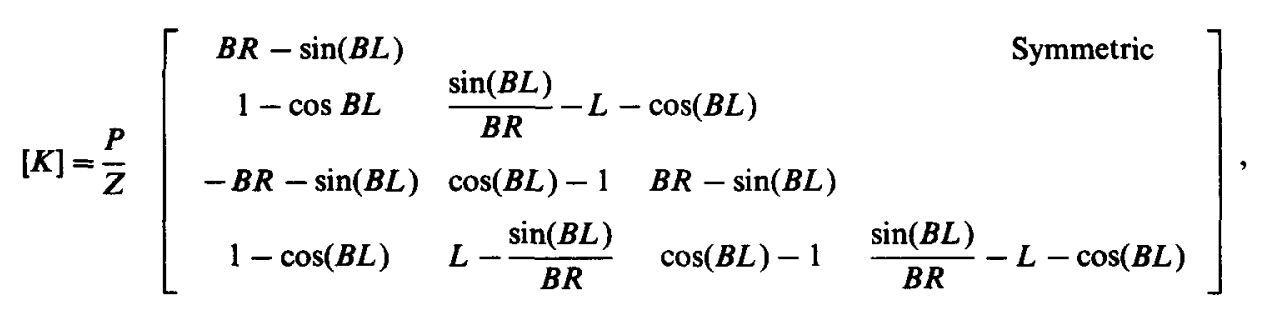


where

$$
\begin{aligned}
& B=\sqrt{ }(P / E I R) \\
& \alpha=B L \\
& R=\left(1-P / K^{\prime} A G\right) \\
& P=\text { axial load }
\end{aligned}
$$

$K^{\prime} A G=$ beam shear rigidity

$I=$ moment of inertia

$z=\sin (B L) \cdot(2 \cdot \tan (B L / 2)-B L R)$.

$$
=\frac{\frac{-P \theta\left(K^{\prime} A G-P\right)}{K^{\prime} A G}}{\frac{P}{K^{\prime} A G}}
$$$$
=\left(P-K^{\prime} A G\right) \theta \text {. }
$$

Thus, Saunders' 'exact' stiffness matrix does not possess the required rigid body rotation mode.

Argyris [11] uses his 'natural formulation' to develop $\left[K_{e}\right]$ and $\left[K_{g}\right]$, which are identical with traditional $\left[K_{e}\right]$ and $\left[K_{g}\right]$. He obtains another matrix $\left[K_{n c}\right]$, referred to as his load correction matrix, which compensates for non-conservative forces.

If we consider the axial load to remain tangent to the slope of the beam at the end points, Argyris' total geometric stiffness matrix $\left[K_{g}+K_{n c}\right]$ becomes

$$
\left[K_{\mathrm{g}}\right]_{\text {TOTAL }}=P\left[\begin{array}{cccccc}
0 & 0 & \sin 2 \beta & 0 & 0 & 0 \\
0 & 6 / 5 L & 1 / 10+\cos 2 \beta & 0 & -6 / 5 L & 1 / 10 \\
0 & 1 / 10 & 2 L / 15 & 0 & -1 / 10 & -L / 30 \\
0 & 0 & 0 & 0 & 0 & -\sin 2 \beta \\
0 & -6 / 5 L & -1 / 10 & 0 & 6 / 5 L & -1 / 10-\cos 2 \beta \\
0 & 1 / 10 & -L / 30 & 0 & -1 / 10 & 2 L / 15
\end{array}\right]
$$

By observation, rigid body translation capability is present in the transverse direction

$$
\begin{aligned}
& \text { Upon multiplying }[K] \cdot\left\{U_{R A R}\right\} \\
& =\frac{P \theta}{z}\left[\begin{array}{c}
-2 \cos (\alpha)-L R \beta \cdot \sin (\alpha)+2 \\
0 \\
2 \cos (\alpha)+L R \beta \cdot \sin (\alpha)-2 \\
0
\end{array}\right]
\end{aligned}
$$

For small $\alpha, \cos (\alpha) \approx 1, \sin (\alpha) \approx \alpha$

$$
\begin{aligned}
-2 \cos (\alpha)-L R \beta \cdot \sin (\alpha)+2 & =-L R \beta \alpha \\
& =-L R B \cdot B L \\
& =-L^{2} R B^{2} \\
\frac{P \theta}{z}= & \frac{P \theta}{\sin (\alpha)(2 \cdot \tan (\alpha / 2)-\alpha R)} \\
\approx & \frac{P \theta}{\alpha(\alpha-\alpha R)} \\
& =\frac{P \theta}{\alpha^{2}(1-R)} .
\end{aligned}
$$

Thus

$$
\begin{aligned}
(P / z)\left(-P L^{2} / E I\right) & =\frac{P \theta\left(-B^{2} L^{2} R\right)}{\alpha^{2}(1-R)} \\
& =\frac{-P \theta R}{(1-R)}
\end{aligned}
$$

The matrix is non-symmetric. Multiplying $\left[K_{g}\right]_{\text {TOTAL }}$ by the exact rigid body rotation vector, then applying small angle considerations, yields $\left[4 \beta^{2}, 0,0,-4 \beta^{2}, 0,0\right]^{T}$, which contains non-zero terms. Hence, $\left[K_{g}\right]_{\text {TOTAL }}$ does not possess rigid body rotation capability. Note that the pseudo-forces now occur in the axial direction.

Martin [12] summarizes work done by Marcal [13] which introduced higher order terms in his initial displacement matrices. In addition to the conventional $\left[K_{e}\right]$ and $\left[K_{g}\right]$, his initial displacement matrices are

$$
\left[\tilde{N}_{1}\right]=A E / L\left[\begin{array}{cccccc}
0 & b_{4} & 0 & 0 & -b_{4} & 0 \\
b_{4} & b_{2} & 0 & -b_{4} & -b_{2} & 0 \\
0 & 0 & 0 & 0 & 0 & 0 \\
0 & -b_{4} & 0 & 0 & b_{4} & 0 \\
-b_{4} & -b_{2} & 0 & b_{4} & b_{2} & 0 \\
0 & 0 & 0 & 0 & 0 & 0
\end{array}\right]
$$

and

$$
\left[\tilde{N}_{2}\right]=P\left[\begin{array}{cccccc}
0 & 0 & 0 & 0 & 0 & 0 \\
0 & 1.5 b_{4}^{2} & 0 & 0 & -1.5 b_{4}^{2} & 0 \\
0 & 0 & 0 & 0 & 0 & 0 \\
0 & 0 & 0 & 0 & 0 & 0 \\
0 & -1.5 b_{4}^{2} & 0 & 0 & 1.5 b_{4}^{2} & 0 \\
0 & 0 & 0 & 0 & 0 & 0
\end{array}\right]
$$

where $u=b_{1}+b_{2} x$ and $v=b_{3}+b_{4} x$.

The basic non-linear equation is

$$
\left[K+1 / 2 \tilde{N}_{1}+1 / 3 \tilde{N}_{2}\right] \cdot\{U\}=\{R\} .
$$




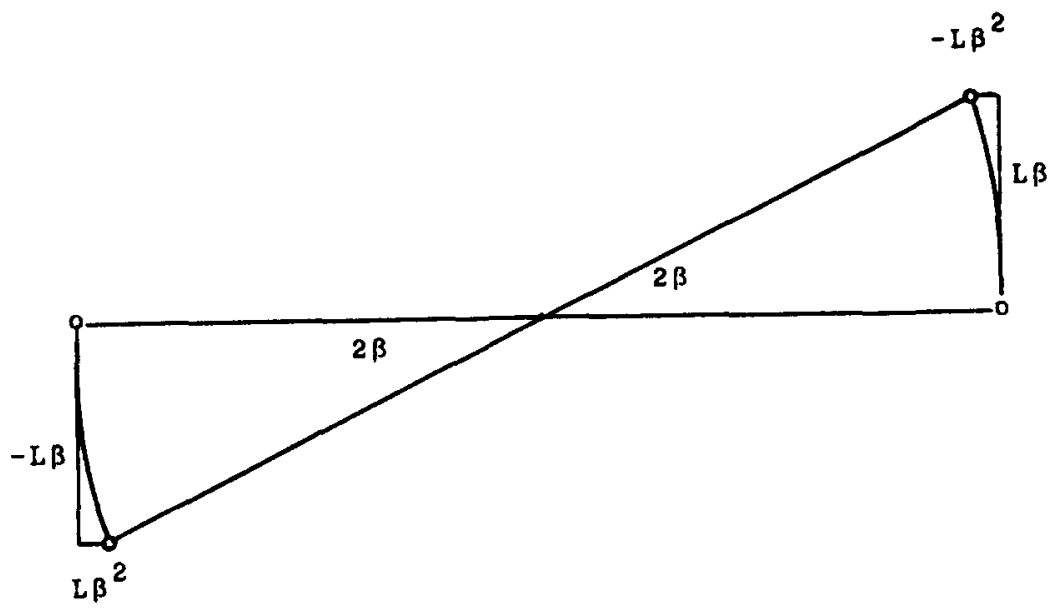

Fig. 2. Rigid body rotation angle of $2 \beta$.

By inspection, $\left[\hat{N}_{1}\right]$ and $\left[\hat{N}_{2}\right]$ possess the required rigid body translation capabilities.

Let the rotation angle $=2 \beta$ (Fig. 2).

$$
\begin{aligned}
u_{1} & =b_{1}, \quad v_{1}=b_{3} \\
u_{2} & =b_{1}+b_{2} L, \quad v_{2}=b_{3}+b_{4} L \\
b_{4} & =\left(v_{2}-v_{1}\right) / L \\
b_{4} & =(L \beta+L \beta) / L \\
b_{4} & =2 \beta \\
b_{4}^{2} / 2 & =4 \beta^{2} / 2=2 \beta^{2} .
\end{aligned}
$$

Similarly

$$
b_{2}=-2 \beta^{2} .
$$

Thus, the rigid body rotation check becomes

$$
\left[K+N_{1}+N_{1}\right] \cdot\left\{U_{R B R}\right\} \text { must equal } 0,
$$

where

$$
\left[N_{1}\right]=A E / L\left[\begin{array}{cccccc}
0 & \beta & 0 & 0 & -\beta & 0 \\
\beta & -\beta^{2} & 0 & -\beta & \beta^{2} & 0 \\
0 & 0 & 0 & 0 & 0 & 0 \\
0 & -\beta & 0 & 0 & \beta & 0 \\
-\beta & \beta^{2} & 0 & \beta & -\beta^{2} & 0 \\
0 & 0 & 0 & 0 & 0 & 0
\end{array}\right]
$$

and

$$
\left[N_{2}\right]=P\left[\begin{array}{cccccc}
0 & 0 & 0 & 0 & 0 & 0 \\
0 & 2 \beta^{2} & 0 & 0 & -2 \beta^{2} & 0 \\
0 & 0 & 0 & 0 & 0 & 0 \\
0 & 0 & 0 & 0 & 0 & 0 \\
0 & -2 \beta^{2} & 0 & 0 & 2 \beta^{2} & 0 \\
0 & 0 & 0 & 0 & 0 & 0
\end{array}\right] .
$$

Performing the rigid body rotation check yields $\left[0,4 A \beta^{3} E-4 \beta^{3} L P-2 \beta P, 0,0\right.$,

$$
\left.-4 A \beta^{3} E+4 \beta^{3} L P+2 \beta P, 0\right]^{T} .
$$

Note that non-zero pseudo-force terms still appear.

Development of the stiffness matrices from the equation of motion has been investigated by $\mathrm{Paz}$, using both a Bernoulli [14] and Timoshenko [15] beam approach. He developed his 'exact' stiffness matrix, then expanded it in a power series solution.

Paz's solution, based on the transverse vibration of a beam with an axial compression load, is of the form

$$
[S]=[K]-\left[G_{0}\right] P-\left[M_{0}\right] \Omega^{2}
$$

$$
-\left[A_{1}\right] P \Omega^{2}-\left[G_{1}\right] P^{2}-\left[M_{1}\right] \Omega^{4} \ldots,
$$

where $[K]$ is the traditional elastic stiffness matrix with no axial terms, $\left[G_{0}\right]$ is the standard geometric stiffness matrix, $\left[M_{0}\right]$ is the first order mass matrix (consistent mass matrix).

$\left[M_{0}\right]=m L / 420$

$$
\times\left[\begin{array}{cccc}
156 & & \text { Symmetric } & \\
22 L & 4 L^{2} & & \\
54 & 13 L & 156 & \\
-13 L & -3 L^{2} & -22 L & 4 L^{2}
\end{array}\right]
$$

$\left[A_{1}\right]$ is the second-order mass-geometrical matrix

$$
\begin{aligned}
{\left[A_{1}\right] } & =m L^{3} / E I \\
\times & {\left[\begin{array}{cccc}
1 / 3150 & & \text { Symmetric } \\
L / 1260 & L^{2} / 3150 & & \\
-1 / 3150 & L / 1680 & 1 / 3150 & \\
-L / 1680 & L^{2} / 3600 & -L / 1260 & L^{2} / 3150
\end{array}\right], }
\end{aligned}
$$


$\left[G_{1}\right]$ is the second-order geometrical matrix

$$
\left[G_{1}\right]=1 / E I \quad\left[\begin{array}{cccc}
L / 700 & & \multicolumn{2}{c}{\text { Symmetric }} \\
L^{2} / 1400 & 11 L^{3} / 6300 & & \\
-L / 700 & -L^{2} / 1400 & L / 700 & \\
L^{2} / 1400 & -13 L^{3} / 12600 & -L^{2} / 1400 & 11 L^{3} / 6300
\end{array}\right]
$$

$\left[M_{1}\right]$ is the second-order mass matrix

$$
\left[M_{1}\right]=\frac{m^{2} L^{5}}{1000 E I}\left[\begin{array}{cccc}
\frac{59}{161 \cdot 7} & & \text { Symmetric } \\
\frac{223 L}{2910 \cdot 6} & \frac{71 L^{2}}{4365 \cdot 9} & & \\
\frac{1279}{3880 \cdot 8} & \frac{1681 L}{23284 \cdot 8} & \frac{59}{161 \cdot 7} & \\
\frac{-1681 L}{2384 \cdot 8} & \frac{-1097 L^{2}}{69854 \cdot 4} & \frac{-223 L}{2910 \cdot 6} & \frac{71 L^{2}}{4365 \cdot 9}
\end{array}\right]
$$

The mass matrices do not possess rigid body modes, but they are not intended to, since they generate the inertial forces. $\left[G_{1}\right]$ possesses all the rigid body modes, hence, no correction to $\left[G_{0}\right]$, which lacks rigid body rotation capability, is applied. Thus, 'grounding' during rigid body rotation still occurs.

Similarly, Paz's Timoshenko formulation (which includes rotary inertia and shear terms), generates the matrix

$$
\begin{aligned}
& {\left[R_{0}\right]=(m L / 30)(R / L)^{2}\left(1+E / K^{\prime} G\right)} \\
& \times\left[\begin{array}{rrrr}
36 & \multicolumn{3}{c}{\text { Symmetric }} \\
3 L & 4 L^{2} & & \\
-36 & -3 L & 36 & \\
3 L & -L^{2} & -3 L & 4 L^{2}
\end{array}\right],
\end{aligned}
$$

where the terms within the matrix are the same as the consistent geometric stiffness matrix. Thus, $\left[R_{0}\right]$ lacks rigid body rotation capabilities.

\section{FORCE UNBALANCE}

Closer examination of the traditional static formulation of $\left[K_{g}\right]$ indicated that there is a load imbalance in the representation, and that pseudo-forces occur to maintain equilibrium [Fig. 3).

Recall that $\left[K_{g}\right]-\left\{U_{R_{B R}}\right\}=\{-P \theta, O, P \theta, O\}$. Using Fig. 3, and letting the sum of the moments at $O$ equal zero, yields

$P L \sin 2 \beta-P^{\prime} L \cos \beta=0$

$$
\begin{aligned}
P^{\prime} & =P-\tan 2 \beta \\
& =P-\tan \theta \\
& =P \theta+\text { higher order terms. }
\end{aligned}
$$

Thus, $P^{\prime}$ represents pseudo-forces required for equilibrium.

Collar and Simpson [16] acknowledge the lack of rigid body rotation capability of $\left[K_{g}\right]$, but indicate that it is not a problem, because the energy representation is correct.

Consider the work/energy relationship from Fig. 3, without $P^{\prime}$.

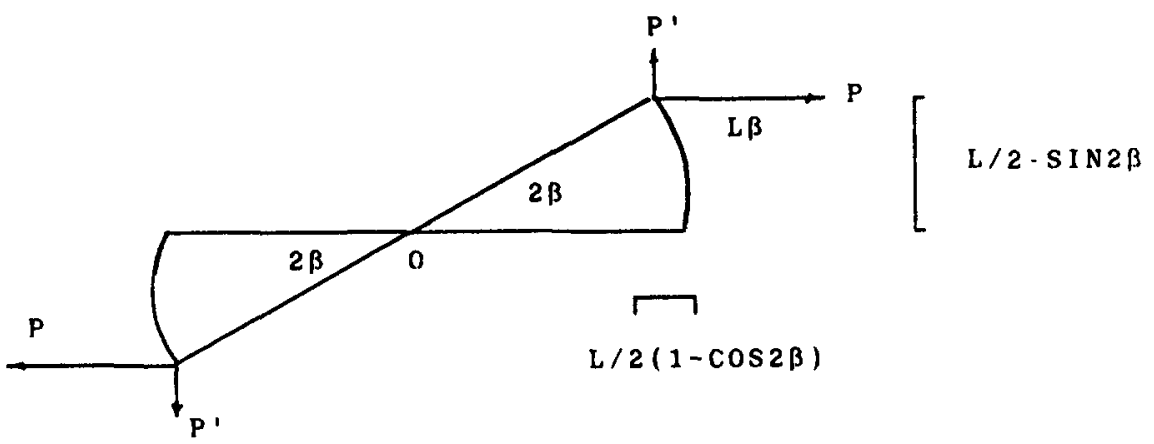

Fig. 3. $P^{\prime}$ represents pseudo-forces required for equilibrium. 


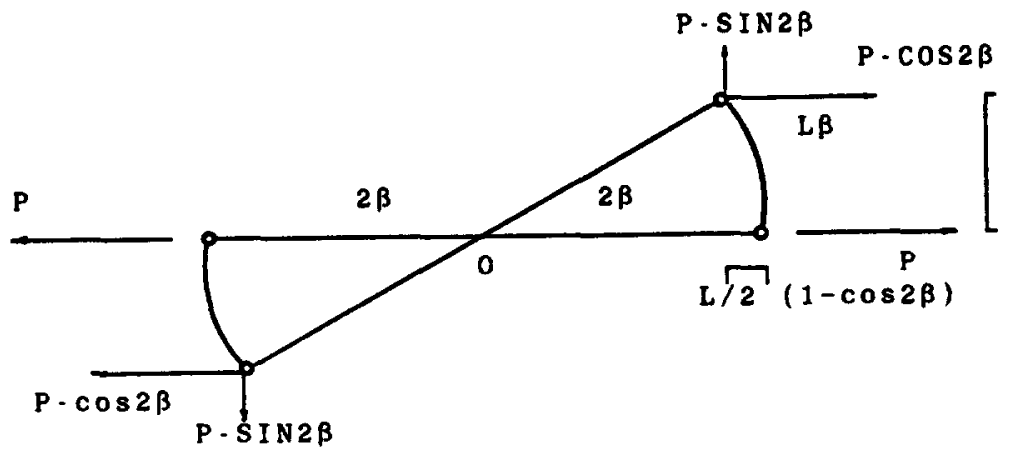

$L / 2$ - S IN $2 \beta$

Fig. 4. Work done during rigid body rotation by follower force.

Work done by $P$

$$
\begin{aligned}
& =P L(1-\cos 2 \beta) \\
& =2 P L(1-\cos 2 \beta) / 2 \\
& =2 P L \cdot \sin ^{2} \beta \\
& =2 P L \beta^{2}+\phi(\beta)^{4}+\text { higher order terms. }
\end{aligned}
$$

Similarly, using a matrix development

$$
\begin{aligned}
\text { Energy } & =1 / 2\{U\}^{T} \cdot[K] \cdot\{U\} \\
& =P \beta^{2} / 2[-2,0,2,0] \cdot[-L, 2, L, 2]^{T} \\
& =2 P L \beta^{2} .
\end{aligned}
$$

Therefore, the energy relationship is correct for the $\beta^{2}$ terms, but the higher order terms are neglected. For large rigid body rotation, this is significant.

It should be noted that as long as the pre-load $P$ is assumed to remain horizontal during rotation, work will be done by the force. Thus, true rigid body rotation cannot occur. In order for the true strain energy to equal zero, the force $P$ must change its orientation as the beam rotates (i.e. a follower force, as in Fig. 4).

Work done $=-L(P+P \cdot \cos 2 \beta)(1-\cos 2 \beta) / 2$

$$
\begin{aligned}
& +P \cdot \sin 2 \beta(L \cdot \sin 2 \beta) / 2 \\
= & P L\left[-(1+\cos 2 \beta)(1-\cos 2 \beta)+\sin ^{2} 2 \beta\right] \\
= & P L / 2\left(-1+\cos ^{2} 2 \beta+\sin ^{2} 2 \beta\right) \\
= & P L / 2(-1+1) \\
= & 0 .
\end{aligned}
$$

\section{SUMMARY}

Based upon this investigation, the following conclusions have been developed:

1. Grounding is due to the development of pseudoforces at the element level required to counteract a force-imbalance inherent in the development. This causes a lack of rigid body rotational capability of the geometric stiffness matrix.

2. Although the consistent geometric stiffness matrix provides acceptable results for most static displacement and buckling problems, provided a sufficient mesh is used, modifications of the global stiffness matrix (zeroing out of erroneous terms, and appending the missing rigid body modes) must be done to more accurately predict the dynamic response.

3. Although the rigid body mode test is routinely used to detect the presence of modeling errors in finite element models, it is not sufficient reason to invalidate a model subjected to pre-loads.

4. Various higher order stiffness matrices developed by others, which include shear and rotatory inertia effects, were examined. As expected, the inclusion of these higher order effects does not compensate for the inaccuracy (lack of rigid body rotation capability) of the geometric stiffness matrix.

5. Development of the geometric stiffness matrix from a directed or follower force approach has the potential for providing full rigid body mode capabilities, since the force imbalance inherent in the other developments can be eliminated.

\section{REFERENCES}

1. K. Carney, J. Chien, D. Ludwiczak, P. Bosela and F. Nekoogar, Photovoltaic Array Modeling and Normal Modes Analysis. NASA Lewis Research Center, Structural Systems Dynamics Branch, Space Station Freedom WP04, Response Simulation and Structural Analysis, September (1989).

2. J. Przemieniecki, Theory of Matrix Structural Analysis. McGraw-Hill (1968).

3. D. Fertis and Chin Lee, Nonlinear vibration and instabilities of elastically supported beams with axial restraints. $A S C E J$. Engng Mech., to be published.

4. P. Bosela, F. Shaker and D. Fertis, Dynamic analysis of space-related linear and non-linear structures. Comput. Struct., to be published.

5. F. Shaker, Effect of axial load on mode shapes and frequencies of beams. NASA TN D-8109 (1975).

6. P. Bosela, NLFINITE.FOR computer program and output (see Appendix).

7. H. Martin, On the derivation of stiffness matrices for the analysis of large deflection and stability problems. AFFDL-TR-66-80. 
8. R. Clough and J. Penzien, Dynamics of Structures. McGraw-Hill (1975).

9. P. Bosela, Development of a 3-node beam element with tension pre-load. Not published.

10. H. Saunders, Stiffness matrix of a beam-column including shear deformation. Shock Vibr. Bull. 40, 187-196 (1969).

11. J. Argyris and Sp. Symeonidis, Nonlinear finite element analysis of elastic systems under non-conservative loading-natural formulation. Part 1. Quasistatic problems. Comput. Meth. appl. Mech. Engng 26, 75-123 (1981).

12. H. Martin, Finite elements and the analysis of geometrically non-linear problems. In Recent Advances in
Matrix Methods of Structural Analysis and Design (Edited by Gallagher, Yamada and Den) (1971).

13. P. Marcal, The effect of initial displacements on problems of large deflection and stability. Brown University, ARPA E54 (1967).

14. M. Paz and L. Dung, Power series expansion of the general stifiness matrix for beam elements. Int. J. Numer. Meth. Engng 9, 449-459 (1975).

15. M. Paz and L. Dung, Power series expansion of the general stiffness matrix including rotary inertia and shear deformation. Shock Vibr. Bull. 46, 181-184 (1976).

16. A. Collar and A. Simpson, Matrices and Engineering Dynamics. Halsted Press, New York (1987).

APPENDIX

NLFINITE,FOR: FULL KE+KG MATRICES, REVISEO 10-24-90

THE AREA ARRAY A IS:

$A(1)=0.4800000 E+02$

THE ELASTICITY ARRAY E IS:

$E(1)=0.3000000 E+08$

THE MOMENT OF INERTIA ARRAY IA IS;

$I A(1)=0.1000000 E+04$

THE GAMMA ARRAY IS:

GAMMA( 1) $=0.3525000 E-01$

THE AXIAL PRETENSION LOAD IS:

0 .

THE JOINT-NUMBER MATRIX IS;

12

THE JOINT COORDINATES ARE;

$X(1)=0.0000000 E+00 \quad Y(1)=0.0000000 E+00$

$X(2)=0.1000000 E+03 \quad Y(2)=0.0000000 E+00$

THE REDUCED SYSTEM STIFFNESS MATRIX IS:

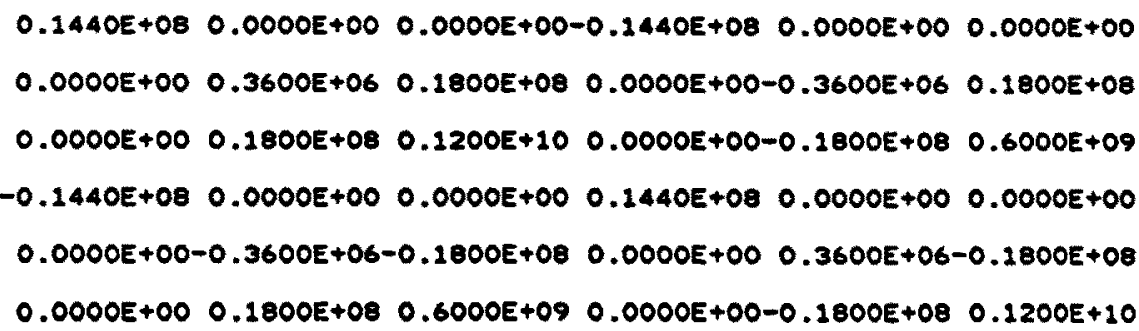

THE REDUCED SYSTEM MASS MATRIX IS:

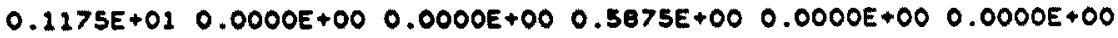

$0.0000 E+00 \quad 0.1309 E+02 \quad 0.1846 E+02 \quad 0.0000 E+00 \quad 0.4532 E+00-0.1091 E+02$

$0.0000 E+00 \quad 0.1846 E+02 \quad 0.3357 E+03 \quad 0.0000 E+00 \quad 0.1091 E+02-0.2518 E+03$

$\begin{array}{lll}0.5875 E+00 \quad 0.0000 E+00 & 0.0000 E+00 \quad 0.1175 E+01 & 0.0000 E+00 \quad 0.0000 E+00\end{array}$

$0.0000 E+00 \quad 0.4532 E+00 \quad 0.1091 E+02 \quad 0.0000 E+00 \quad 0.1309 E+01-0.1846 E+02$

$0.0000 E+00-0.1091 E+02-0.2518 E+03 \quad 0.0000 E+00-0.1846 E+02 \quad 0.3357 E+03$ 


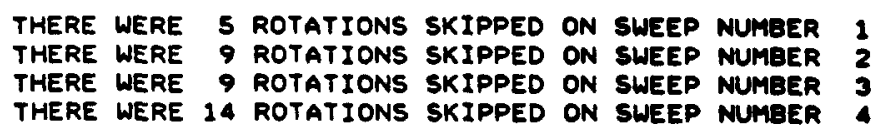

THERE WERE 15 ROTATIONS SKIPPED ON SWEEP MUMBER 5

THE SCALAR PRODUCT OF THE FIRST AND LAST

EIGENVECTORS OF THE TRANSFORMED MATRIX IS 0.00000000000000000

THERE WERE 5 SWEEPS PERFORMED.

THE EIGENYALUES AND EIGENVECTORS FOLLOW:

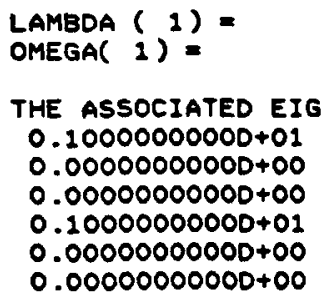
$0.10000000000+01$
$0.00000000000+00$
$0.00000000000+00$
$0.10000000000+01$
$0.00000000000+00$
$0.00000000000+00$

THE ASSOCIATED EIGENVECTOR IS:

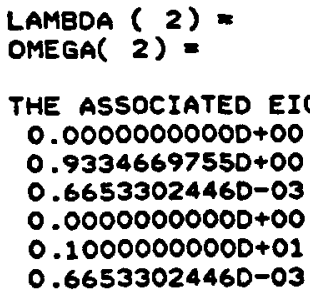

LAMBDA $(3)=$ 0.0000

OMEGA( 3) =

0.0000 RAD/S

THE ASSOCIATED EIGENVECTOR IS:

$0.00000000000+00$

$0.10000000000+01$

$-0.19773193200-01$

$0.00000000000+00$

$-0.97731932040+00$

$-0.1977319320 D-01$

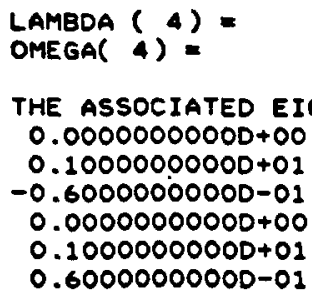

$0.00000000000+00$

$0.10000000000+01$

$-0.60000000000-01$

$0.00000000000+00$

$0.10000000000+01$

$0.60000000000-01$

THE ASSOCIATED EIGENVECTOR IS:

$\begin{array}{lc}\text { LAMBDA }(5)= & 49021276.5957 \\ \text { OMEGA } 5)= & 7002.5196 \text { RAO/S }\end{array}$

THE ASSOCIATED EIGENVECTOR IS:

$0.10000000000+01$

$0.00000000000+00$

$0.00000000000+00$

$-0.10000000000+01$

$0.00000000000+00$

$0.00000000000+00$

LAMBDA $(6)=$ OMEGA 6 ) =

THE ASSOCIATED EIGENVECTOR IS:

$0.0000000000 D+00$

$0.10000000000+01$

$-0.12000000000+00$

$0.00000000000+00$

$-0.10000000000+01$

$-0.12000000000+00$ 